\title{
Diagnosis Value of Procalcitonin Variation on Early Pneumonia after Adult Cardiac Surgery
}

\author{
Hua Jin*, ${ }^{1}$ Su-Ping Gu ${ }^{1}$ Yan Wang, ${ }^{1}$ Ke Pan,${ }^{2}$ Zhong Chen, ${ }^{3}$ Hai-Long Cao, ${ }^{1}$ Dong-Jin Wang ${ }^{1,2,3}$ \\ ${ }^{1}$ Department of Cardio-Thoracic Surgery, Nanjing Drum Tower Hospital, The Affiliated Hospital of Nanjing University Medical \\ School, Nanjing, Jiangsu, China; ${ }^{2}$ Nanjing Drum Tower Hospital, the Affiliated Clinical College of Xuzhou Medical University, \\ Nanjing, Jiangsu, China; ${ }^{3}$ Department of Cardio-Thoracic Surgery, Nanjing Drum Tower Hospital, Nanjing Medical University, \\ Nanjing, Jiangsu, China
}

\section{ABSTRACT}

Background: Postoperative pneumonia (PP) is a complication after cardiac surgery. This study aimed to investigate the ability of procalcitonin (PCT) variation to diagnose postoperative pneumonia.

Method: In this prospective observational study, patients with PP and age- and sex-matched cases in our center from October 10, 2020, to January 31, 2021, were included. Patients diagnosed with PP in this study met both clinical and microbiological diagnostic criteria. Blood samples were collected in all patients from the first postoperative day (POD1) to POD5 to measure PCT, white blood cells (WBCs), and C-reactive protein (CRP). PCT variation was calculated by the equation: (PCTdelayed - PCTPOD1)/PCTPOD1. The receiver operating characteristic and area under the curve (AUC) analyses were used to evaluate the diagnostic performance of different biomarkers.

Results: Our study enrolled 272 patients, including 24 patients with PP and 248 age- and sex-matched cases. From POD1 to POD5, the absolute value of PCT showed diagnostic significance for pneumonia $(P<.05), \mathrm{WBC}$ showed no differences, and CRP had no diagnostic value until POD4. Furthermore, PCT variation showed the best diagnostic value among those biomarkers (AUC $0.84,95 \%$ confidence interval [CI] 0.71, 0.91). Multivariable logistic regression showed that PCT variation on POD2 had significant value to predict $\mathrm{PP}$ (odds ratio 5.602, 95\% CI 2.178, 14.409, $P<.01$ ).

Conclusion: Compared with PCT level, WBC count, and CRP level, PCT variation had the best diagnostic value in predicting PP.

Received May 22, 2021; accepted Fune 16, 2021.

*Hua fin and Su-Ping Gu contributed equally to this work.

Correspondence: Dr. Dong-fin Wang or Dr. Hai-Long Cao, Cardio-Thoracic Surgery, Nanjing Drum Tower Hospital, The Affiliated Hospital of Nanjing University Medical School, Number 321 Zhongshan Road, Nanjing, Fiangsu, 210008, China; (e-mail: dongiin_wang@126.com [D-f. Wang] or 13675186233@163.com [H-L.Cao]).

\section{INTRODUCTION}

Postoperative pneumonia (PP) is a serious complication after cardiac surgery with cardiopulmonary bypass (CPB) [Kinlin 2010]. Mortality, duration of hospital stay, and costs significantly increased in patients with cardiac surgery complicated by PP [Kinlin 2010; Torres 2017; Jiang 2018; He 2014]. Early diagnosis can effectively improve the prognosis of those patients [Wallgren 2016; Weiss 2014]. Therefore, it is very important to find a biomarker that can diagnose $\mathrm{PP}$ early.

Levels of procalcitonin (PCT), white blood cell (WBCs), and $\mathrm{C}$-reactive protein (CRP) are routinely used to predict postoperative infections in clinical practices [Lee 2017; Russell 2019; Hu 2017]. However, which of these biomarkers can best diagnose PP is controversial. Some studies have reported that PCT had better diagnostic performance than WBC count or CRP in patients with infections [Tan 2019; Li 2017]. However, the clinical symptoms of PP in the early stages usually overlap with noninfectious systemic inflammatory response syndrome (SIRS) occurring after cardiac surgery [Jukica 2015; Heredia-Rodríguez 2017; Pittet 1995]. Because PCT is one of the inflammatory mediators involved in SIRS and will significantly increase after cardiac surgery [He 2014; Wallgren 2016; Weiss 2014], high levels may mislead clinicians to predict PP in patients undergoing open-heart surgery. A previous study reported that continuously detected PCT might be more helpful to diagnose infections [Spaziante 2018]. Meanwhile, Li et al [2017] postulated that PCT variation could diagnose infections early after pediatric heart surgery compared with the absolute value of PCT, WBC count, or CRP. Unfortunately, none of these measurements can effectively diagnose PP in the first few days after adult cardiac surgery. We therefore designed this study to evaluate the value of PCT variation, absolute PCT, WBC count, and CRP for the early diagnosis of PP.

\section{METHODS}

The authors declare that all methods were carried out in accordance with relevant guidelines and regulations. The datasets used during the current study are available from the corresponding author on reasonable request. 


\section{Study Design and Settings}

This work was based on prospective observational research. Ethics approval was obtained from the Medical Ethics Committee of Affiliated Nanjing Drum Tower Hospital, Nanjing University Medical College (2020-249-01), and the study was conducted according to the principles in the Declaration of Helsinki. Patients, or their relatives when patients could not consent, provided written informed consent to participate in the study.

We included consecutive patients with PP and matched the controls at a ratio of $1: 10$ by age \pm 3 years and sex in our center from October 10, 2020, to January 31, 2021. A total of 272 patients met the eligibility criteria, including 24 $\mathrm{PP}$ patients (PP group) and 248 age- and sex-matched cases (non-PP group). Inclusion criteria were shown as follows: (1) adult patients (18 to 80 years old) undergoing cardiac surgery with CPB; (2) written informed consent to participate in the study. Exclusion criteria were (1) preoperative body temperature $\geq 38^{\circ} \mathrm{C}, \mathrm{WBCs}>10,000$ cells $/ \mathrm{mm}^{3}$, or serum PCT $>0.5$ $\mathrm{ng} / \mathrm{mL}$; (2) patients undergoing cardiac surgery for trauma, infective endocarditis, neoplasms, and malignant tumors; (3) patients diagnosed with any other infectious diseases (surgical site infection, sepsis, etc.); (4) patients diagnosed with inflammatory immune diseases or connective tissue diseases; (5) pregnant or lactating women; and 6) patients with missing clinical data (because of perioperative death or other reasons).

\section{$C P B$ and Perioperative Management}

All surgical operations were performed through a median sternotomy. CPB was used in all patients. The ascending aorta was cannulated with a size-appropriate cannula. Venous cannulations were chosen with separate cannulas in the superior and inferior vena cava. The initial volume of the antegrade cold blood cardioplegia solution (4:1 cardioplegia solution to blood ratio) was twice the volume needed for the cessation of all cardiac electrical activity but never $<1,000 \mathrm{~mL}$. Cardiac arrest was maintained by the retrograde infusion of $300 \mathrm{~mL}$ of blood cardioplegia solution (8:1 cardioplegia solution to blood ratio) every 20 minutes. Occasionally, intermittent antegrade cold blood cardioplegia was used according to the surgeon's preferences. Prophylactic antibiotic therapy was given routinely in the perioperative period to prevent infection.

The first dose of antibiotics was injected intravenously within 60 minutes of the incision. Successive doses were administered every 3 to $4 \mathrm{~h}$ during surgery [Engelman 2007]. In addition, we continued to administer adequate antibiotics in the first 48 hours after the operation per guidelines. We routinely choose first-generation cephalosporins as prophylactic intravenous antibiotics and use vancomycin when the clinician believes it necessary [Engelman 2007; Edwards 2006; Vos 2018].

\section{Biologic Measurements}

Blood samples for measurement of serum concentrations of PCT, CRP, and WBCs were obtained separately before surgery and daily until the fifth postoperative day (POD5). PCT was measured with an immunoluminometric assay whose detection limit ranged from 0.05 to $25 \mathrm{ng} / \mathrm{mL}$; normal value was $<0.5 \mathrm{ng} / \mathrm{mL}$. CRP was measured by automatic laser nephelometry; normal value was $<8 \mathrm{mg} / \mathrm{L}$. WBC count was determined with an automatic counter; normal value was 4,000 to 10,000 cells $/ \mathrm{mm}^{3}$. The coefficient of variation of the measurements was $<5 \%$. Patients underwent sputum culture with fiberoptic bronchoscope or alveolar lavage fluid at least twice in the first 5 days after surgery and, if necessary, a pleural effusion culture examination to find pathogenic bacteria.

\section{Reference Diagnostic Criteria}

$\mathrm{PP}$ was defined when microorganisms were isolated from bronchial secretions at least twice (the first microorganism obtained on POD2) and the following clinical criteria were met [Bone 1992; Calandra 2005]: (1) $\geq 1$ of the following symptoms: purulent sputum, auscultation findings suspicious of pneumonia or hypoxemia, or body temperature $>38^{\circ} \mathrm{C}$; and (2) infiltrate on chest radiograph.

The final diagnosis of PP was determined by 2 independent experts in regard to the complete medical chart. In cases of disagreement, a third expert helped reach a consensus. Each final diagnosis was classified as microbiologically confirmed, probable, possible, or absent [Calandra 1992]. The final diagnosis was reached when it was classified as microbiologically confirmed by the experts and ruled out when the experts classified it as absent, probable, or possible. Experts were blinded to PCT, CRP, and WBC count. Finally, patients were divided into 2 groups: the non-PP group, who did not develop postoperative infection within 5 days after surgery; and the PP group, who developed pneumonia within 5 days after surgery.

\section{Data Collection}

We collected demographic characteristics, preoperative diagnosis, surgical methods, extracorporeal circulation time, ascending aorta occlusion time, mechanical ventilation time, intensive care unit time, hospital stay duration, imaging examination results, and microbiological examination results through the electronic case system. Serum PCT, CRP, and WBC concentrations were also recorded daily until POD5. PCT variation was defined by the equation (PCTdelayed PCTPOD1)/PCTPOD1. PCTPOD1 is the value of PCT measured on POD1; PCTdelayed is blood PCT concentration measured on POD2 to POD5. A negative value indicates a decrease in PCT concentration.

\section{Sample Size}

In this study, we evaluated the diagnostic value of biomarkers based on receiver operating characteristic (ROC) curves and area under the curve (AUC). Therefore, we calculated the sample size required for the study based on AUC using Power Analysis and Sample Size (version 15.0). In previous studies, the AUC of PCT to diagnose infection ranged from approximately 0.81 to $0.90[\mathrm{Li}$ 2017; Klingele 2016]. Therefore, we set the AUC of this study to 0.85 . Considering a 2 -sided significance level of $5 \%(\alpha)$ and a test power of $80 \%(1-\beta), 24$ patients with postoperative pneumonia would be required. Because we have a 1:10 age and sex match, our control group needed at least 240 patients.

\section{Statistic Analysis}

IBM SPSS statistical software was used (Statistics for Windows, version 26, IBM Corp., Armonk, NY). Normally 
distributed variables were expressed as mean \pm standard deviation and compared using Student's t test. Nonparametric continuous variables were expressed as median (interquartile range [IQR]) and compared using Mann-Whitney $U$ test. Continuous variables were determined to be normal in distribution by Shapiro-Wilk test. Categorical data were equated using $\chi^{2}$ test or Fisher's exact test.

We used ROC curves and AUC to evaluate the value of WBCs, CRP, PCT, and PCT variation in diagnosing PP. For infection prediction, cutoff values for WBCs, CRP, and PCT were chosen to correspond to the best respective Youden's index calculated as follows: Youden's index $=$ sensitivity + specificity - 1. Results are expressed for AUC as mean and 95\% confidence interval (CI). For additional analyses, all covariates (previous cardiac surgery; $\mathrm{CPB}$ time; ascending aortic occlusion time; mechanical ventilation time; intensive care unit stay duration; hospital stay duration; CRP on POD1, POD4, and POD5; PCT on POD1, POD2, POD3, and POD5; and PCT variation on POD2 to POD5) in univariate modeling were entered into a multivariable logistic regression model, with the likelihood ratio test for forward selection designed to assess the independently associated postoperative factors with PP. Collinearity diagnostics were performed using tolerance estimates for individual variables in a linear regression model. The 2 groups were analyzed using repeatmeasures analysis of variance (ANOVA). Differences between 2 groups were determined by repeat-measures ANOVA with subsequent Bonferroni correction, with $P<.05 / \mathrm{n}$ considered significant. All reported $P$ values are 2 sided, and values of $P<$ .05 were considered to indicate statistical significance.

Table 1. Clinical Baseline Data

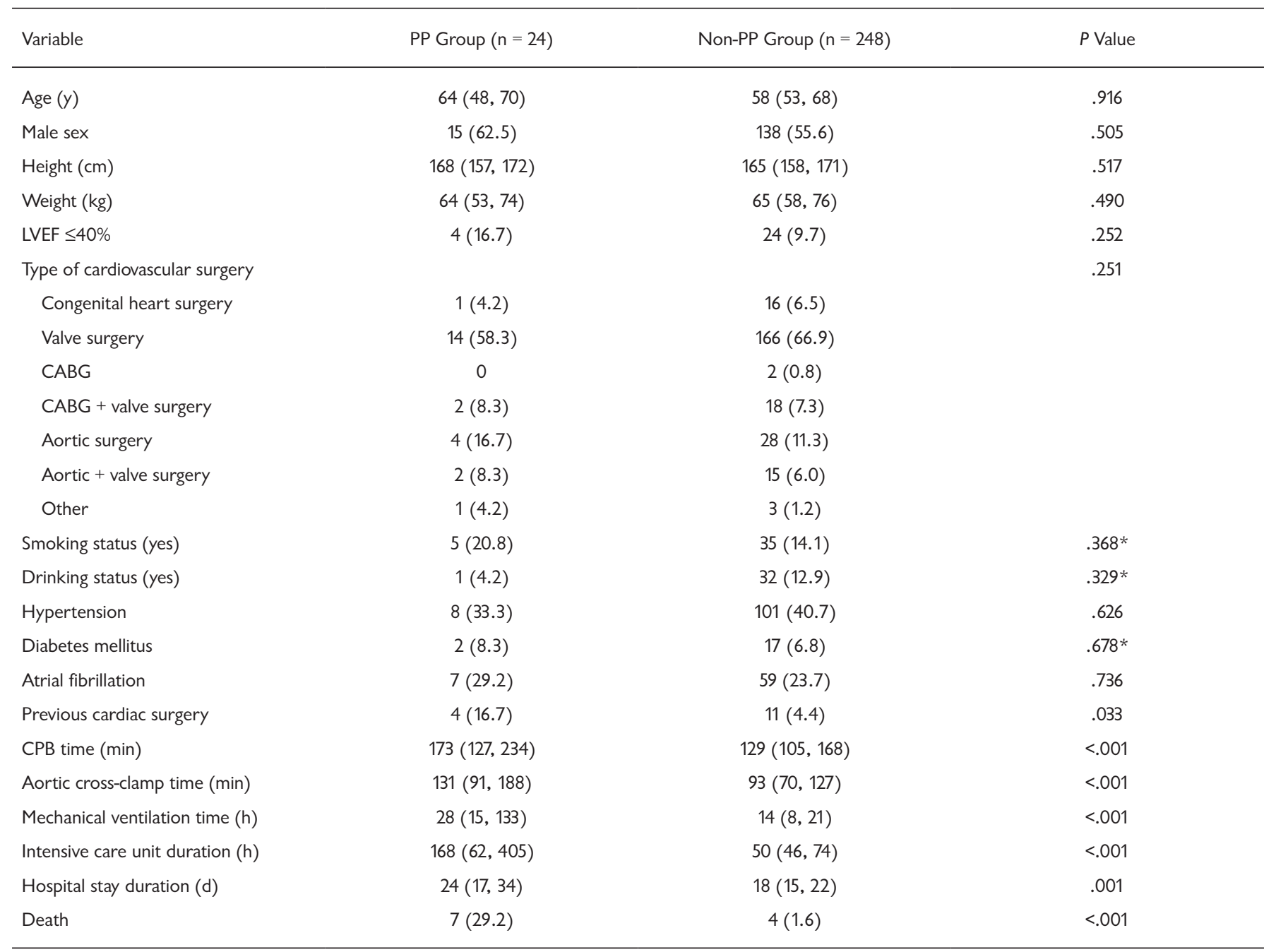

Data are median (interquartile range) or $\mathrm{n}(\%)$.

*Fisher's exact test.

CABG indicates coronary artery bypass grafting; LVEF, left ventricular ejection fraction. 
RESULTS

\section{Patient Characteristics}

The study included 272 age- and sex-matched patients, of whom 24 were diagnosed with PP (PP group) and 248 without infections were categorized into the non-PP group. No significant differences were found regarding age, sex, height, weight, left ventricular ejection fraction, type of cardiac surgery, smoking status, drinking status, hypertension, diabetes mellitus, or atrial fibrillation. The proportion of patients in the PP group with a history of cardiac surgery was higher (16.7\% versus $4.4 \%, P=.03$ ). Longer $\mathrm{CPB}$ time and ascending aortic occlusion time were significantly related to the occurrence of PP $(P<.01)$. Patients in the PP group used mechanical ventilation for a longer time $(P<.01)$, were in intensive care for longer $(P<.01)$, and had longer hospital stays $(P=.01)$ (Table 1$)$.
WBCs, CRP, PCT, and the Value of PCT Variation in PP and Non-PP Patients

There were no significant differences in PCT (median 0.04, IQR 0.04 to $0.06 \mathrm{ng} / \mathrm{mL}$ versus median 0.04 , IQR 0.04 to 0.04 $\mathrm{ng} / \mathrm{mL}, P=.139$ ), WBCs (median 5.9 , IQR 4.5 to $6.6 \times 109 / \mathrm{L}$ versus median 5.8, IQR 4.7 to $6.9 \times 109 / \mathrm{L}, P=.659)$, or $\mathrm{CRP}$ (median 0.58 , IQR 0.02 to $2.12 \mathrm{mg} / \mathrm{L}$ versus median 0.65 , IQR 0.28 to $1.85 \mathrm{mg} / \mathrm{L}, P=.769$ ) before surgery between the 2 groups (Table 2). (Patients with WBCs $\geq 10 \times 109 / \mathrm{L}$ or PCT $>0.5 \mathrm{ng} / \mathrm{mL}$ before surgery were excluded.)

Serum PCT in the PP group continued to rise for the first 2 days after surgery, and the PP group had significantly higher PCT levels compared with the non-PP group $(P<.01)$ (Fig. 1). After Bonferroni correction, the $\mathrm{PP}$ group had higher PCT during POD2 to POD5 $(P<.05 / \mathrm{n}=5)$. WBCs peaked on POD2 and showed no difference between the 2 groups from POD1 to $\operatorname{POD} 5(P<.01)$ (Fig. 2); after Bonferroni

Table 2. Serum Procalcitonin Concentrations and Procalcitonin Variation

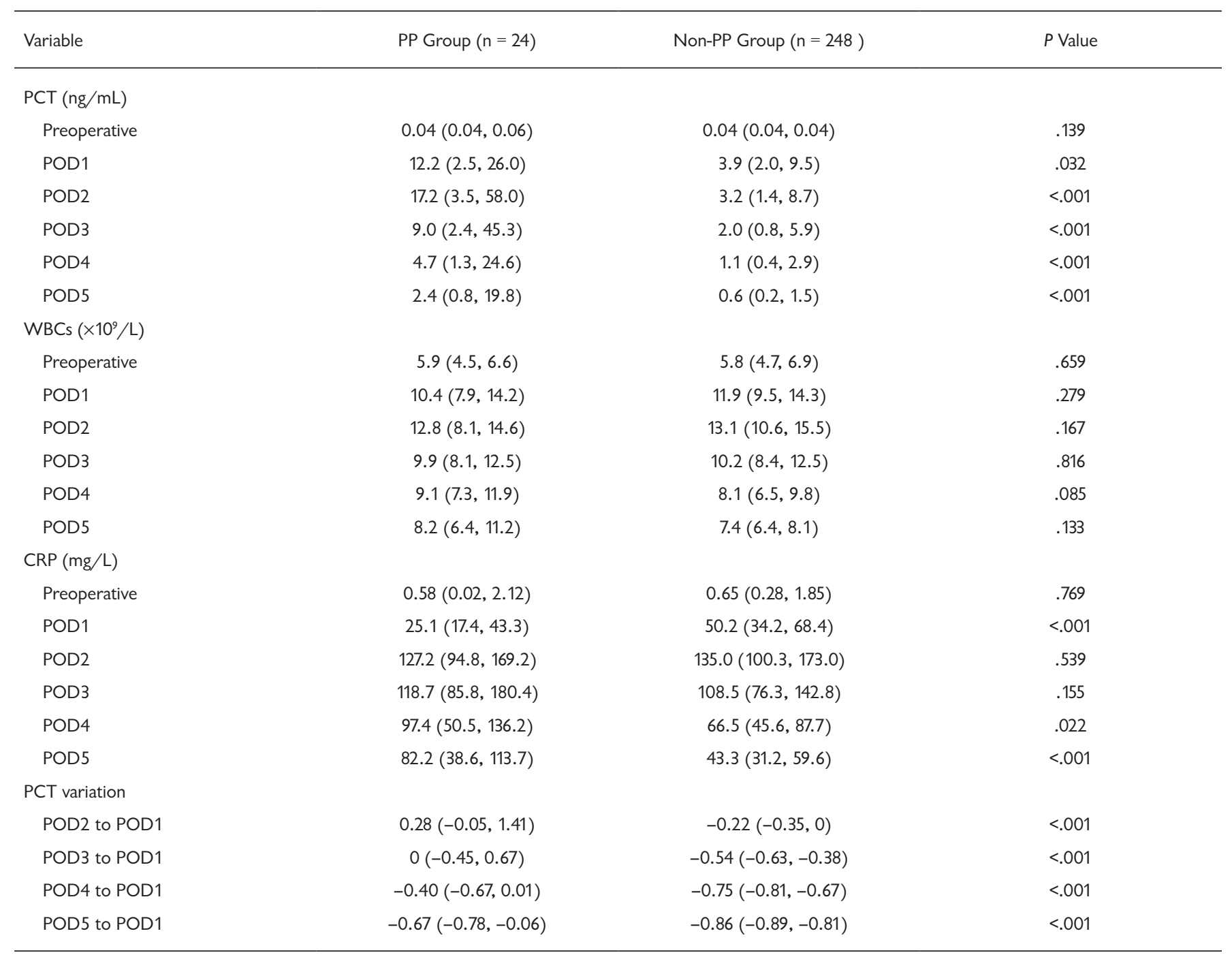

Data are median (interquartile range). 
correction, there are no differences between the groups ( $P$ $>.05 / \mathrm{n}=5$ ). From POD1 to POD5, the CRP level of the PP group was higher than that of the non-PP group $(P<.01)$ (Fig. 3). After Bonferroni correction, CRP showed significant differences between the groups on POD1 and POD5 $(P<$ $.05 / \mathrm{n}=5)$. In the PP group, CRP levels were lower on POD1 $(P<.01)$.

\section{Diagnostic Value of WBCs, CRP, PCT, and PCT Variation}

ROC curves showed that the AUCs of PCT on POD2 to POD5 and PCT variation from POD2 to POD5 were $>0.7$ (Table 3). The PCT variation had the best diagnostic value
(AUC >0.8) (Fig. 4; Table 3). In particular, PCT variation on POD2 predicted PP with an AUC of 0.81 (95\% CI 0.70, 0.91, cutoff -0.08 , sensitivity 0.83 , specificity 0.67 ). Detailed data are shown in Table 3.

\section{Multivariate Analysis Between PP and Non-PP Groups}

Multivariable logistic regression showed that PCT on POD 5, PCT variation on POD 2, aortic cross-clamp time, and duration of hospital stay were independently correlated with PP. Among them, PCT variation on POD2 was the strongest risk factor for PP (odds ratio $5.602,95 \%$ CI 2.178 , 14.409) (Table 4).

Table 3. Comparison of AUC and Efficiency of WBC, CRP, and PCT Levels and PCT Variation in the Diagnosis of Postoperative Infection

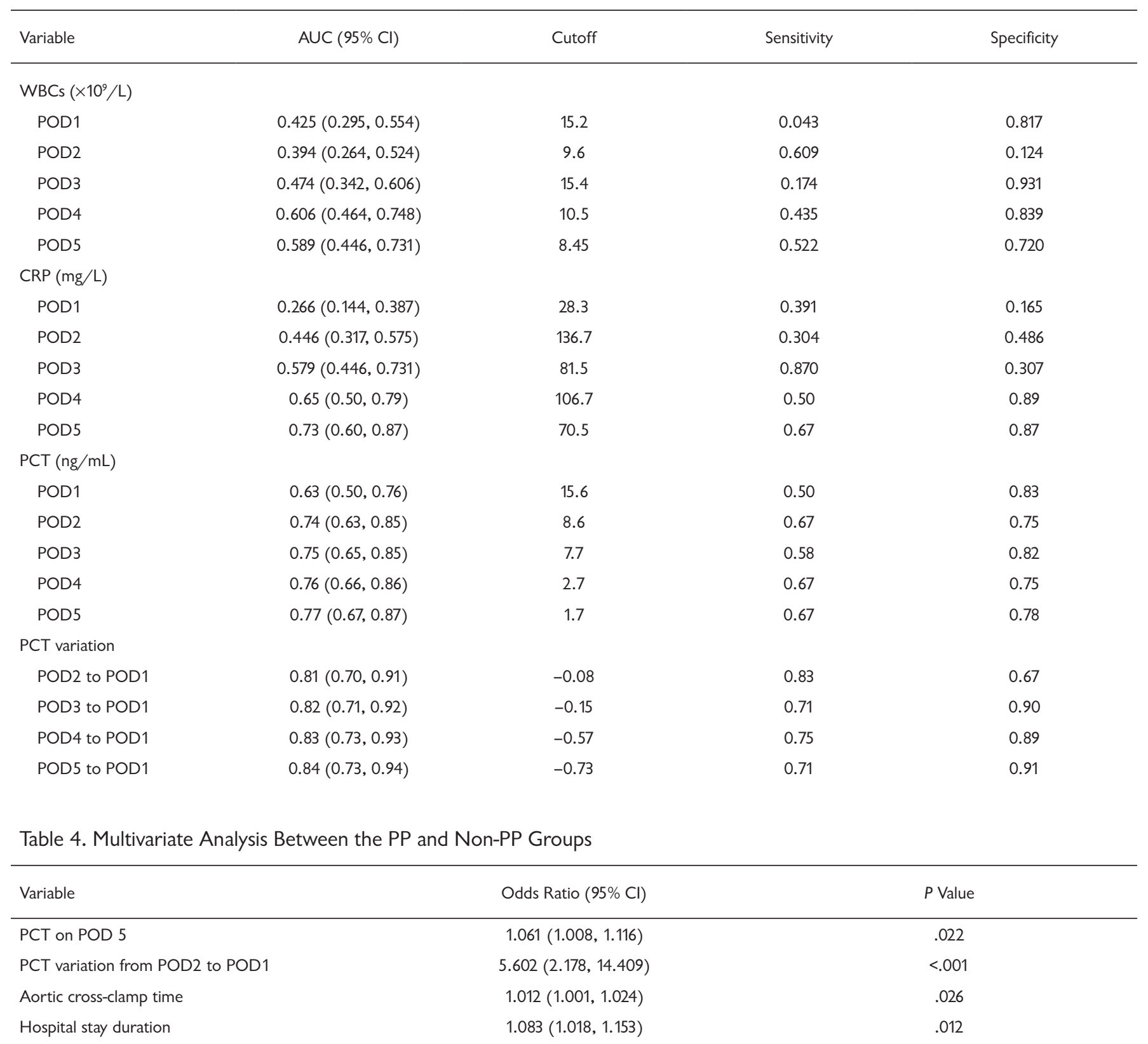




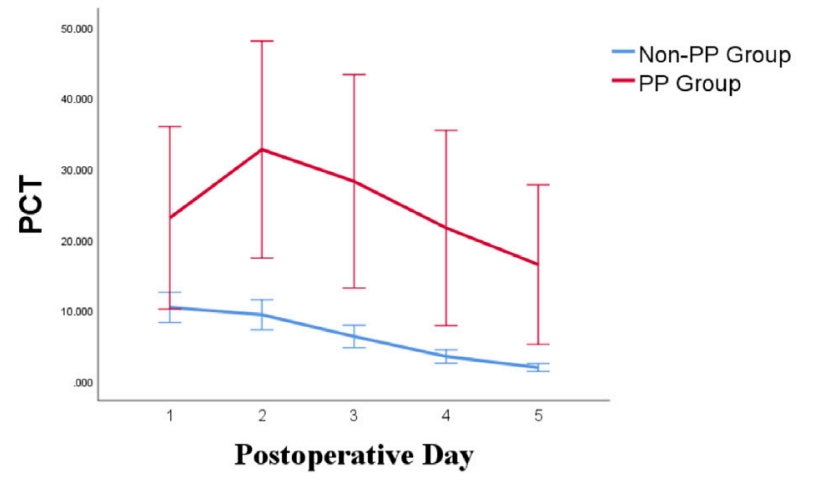

Figure 1. PCT levels from POD1 to POD5 (error bar graph: 95\% Cl).

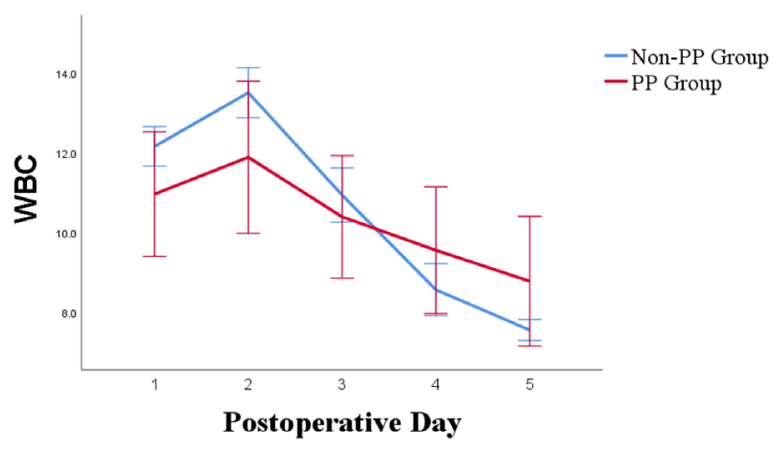

Figure 2. WBC levels from POD1 to POD5 (error bar graph: 95\% Cl).

\section{DISCUSSION}

There is no doubt that PCT can help diagnose infections more accurately than WBC and CRP [Li 2017]. PCT variation may be even more useful and helpful to predict PP compared with the absolute value after pediatric cardiac surgery [Li 2017]. However, there was a lack of evidence that PCT variation or PCT could be used to predict PP in adult cardiac patients. Therefore, it was necessary to further verify the diagnostic value of PCT variation. In our study, we found that PCT variation and PCT could be more effective than WBCs and CRP in diagnosing PP. Moreover, PCT variation had better diagnostic performance compared with PCT levels. We suggest that PCT variation could be used to predict PP earlier in adult cardiac patients.

After CPB, an inflammatory cascade may occur, very similar to that observed in sepsis [Aouifi 1999]. It is related to the exposure of plasma proteases to the nonendothelial inner membrane of the CPB circuit. At the same time, ischemiareperfusion injury during $\mathrm{CPB}$ is also an inflammatory phenomenon, which is another important cause of postoperative morbidity [Bronicki 2016]. Previous studies had shown that clinical manifestations (fever, tachycardia, etc.), and traditional biomarkers (leukocytosis) are not reliable for differential diagnosis of infected and noninfected SIRS after CPB [Pittet 1995]. Our study indicated that patients had high levels of WBCs, CRP, and PCT after cardiac surgery, and it

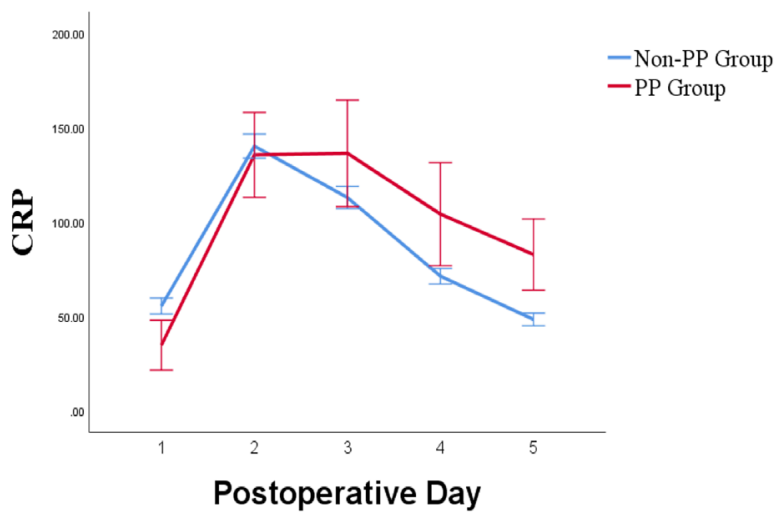

Figure 3. CRP levels from POD1 to POD5 (error bar graph: 95\% Cl).

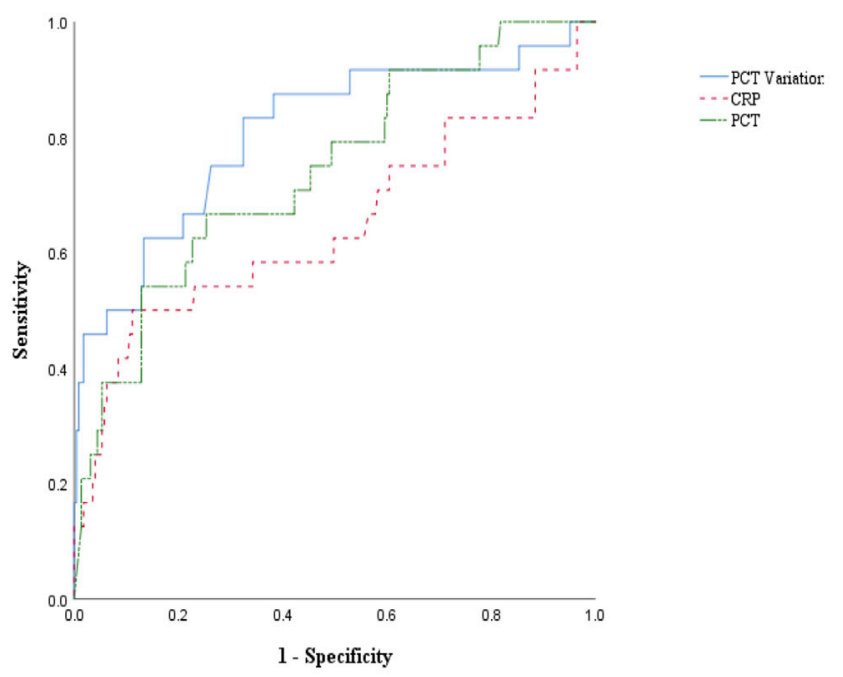

Figure 4. Comparison of ROC curves showing the predictive value of PCT (value on POD2), PCT variation (value on POD2), and CRP (value on POD4) for the diagnosis of postoperative pneumonia.

may reconfirm that SIRS frequently occurred in patients who underwent cardiac surgery. Our study found that patients with PP had higher PCT levels compared with patients without PP from POD2 to POD5, and WBCs and CRP in the PP group had no significant differences compared with the non-PP group (Figs. 1 to 3). This may suggest that PCT and PCT variation had better predictive power for PP. Meanwhile, PP variation had the best diagnostic ability among these biomarkers. Therefore, the diagnosis of $\mathrm{PP}$ could occur earlier with continuous PCT monitoring, allowing earlier administration of antibiotics in patients with PP.

The best timing of diagnosis of PCT in infection after CPB is still controversial. $\mathrm{Li}$ et al [2017] reported that the PCT had best sensitivity and specificity on POD7. Aouifi et al [1999] showed that PCT was reliable for diagnosis of infection on POD2 (sensitivity 0.85 , specificity 0.95 ), with a cutoff value of $1 \mathrm{ng} / \mathrm{mL}$. However, Aouifi's study included just 36 patients. In our study, the PCT level of the PP group was 
significantly higher than that of the non-PP group on POD2. To be diagnosed with PP in our study, patients had to have microorganisms isolated at least twice. Thus our study used a more accurate standard than previous studies, which might be why PCT variation could predict PP earlier. In general, PCT variation has better diagnostic performance than the other biomarkers studied.

\section{Limitations}

Some limitations should be considered in this study. First, this is an observational analysis, and the results support association, not necessarily causation. Second, the data come from a single center, and agency-specific variables may have influenced the results. Therefore, our results must be further validated by other multicenter studies. How to improve the generalization and practicability of the research results will be the direction of our future research.

\section{Conclusions}

PCT variation showed better diagnostic performance than serum PCT, WBCs, or CRP in patients after cardiac surgery with CPB.

\section{ACKNOWLEDGMENTS}

This work was supported by Jiangsu Provincial Key Medical Discipline of the Project of Invigorating Health Care through Science, Technology and Education, grant number ZDXKA2016019.

\section{REFERENCES}

Aouifi A, Piriou V, Blanc P, et al. Effect of cardiopulmonary bypass on serum procalcitonin and C-reactive protein concentrations. Br J Anaesth 1999;83:602-607.

Bone RC, Balk RA, Cerra FB, et al. Definitions for sepsis and organ failure and guidelines for the use of innovative therapies in sepsis. The ACCP/SCCM Consensus Conference Committee. American College of Chest Physicians/Society of Crit Care Med. Chest 1992;101:1644-1655.

Bronicki RA, Hall M. Cardiopulmonary bypass-induced inflammatory response. Pediatr Crit Care Med 2016;17:S272-S278.

Calandra T, Cohen J. The International Sepsis Forum Consensus Conference on Definitions of Infection in the Intensive Care Unit. Crit Care Med 2005;33:1538-1548.

Edwards FH, Engelman RM, Houck P, Shahian DM, Bridges CR, Society of Thoracic Surgeons. The Society of Thoracic Surgeons Practice Guideline Series: Antibiotic prophylaxis in cardiac surgery, Part I: Duration. Ann Thorac Surg 2006;81:397-404.

Engelman R, Shahian D, Shemin R, et al. The Society of Thoracic Surgeons practice guideline series: Antibiotic prophylaxis in cardiac surgery, part II: Antibiotic choice. Ann Thorac Surg 2007;83:1569-1576.

He S, Chen B, Li W, et al. Ventilator-associated pneumonia after cardiac surgery: a meta-analysis and systematic review. J Thorac Cardiovasc Surg 2014;148:3148-3155.e1-5.
Heredia-Rodríguez M, Bustamante-Munguira J, Lorenzo M, et al. Procalcitonin and white blood cells, combined predictors of infection in cardiac surgery patients. J Surg Res 2017;212:187-194.

Hu L, Shi. Q, Shi M, Liu R, Wang C. Diagnostic Value of PCT and CRP for detecting serious bacterial infections in patients with fever of unknown origin: a systematic review and meta-analysis. Appl Immunohistochem Mol Morphol 2017;25:e61-e69.

Jiang WL, Hu XP, Hu ZP, Tang Z, Wu HB, Chen LH, et al. Morbidity and mortality of nosocomial infection after cardiovascular surgery: a report of 1606 cases. Curr Med Sci 2018;38:329-335.

Jukica. T, Ihanb. A, Stubljar. D. Dynamics of inflammation biomarkers C-reactive protein, leukocytes, neutrophils, and CD64 on neutrophils before and after major surgical procedures to recognize potential postoperative infection. Scand J Clin Lab Invest 2015;75:500-507.

Kinlin LM, Kirchner C, Zhang H, Daley J, Fisman DN. Derivation and validation of a clinical prediction rule for nosocomial pneumonia after coronary artery bypass graft surgery. Clin Infect Dis 2010;50:493-501.

Klingele M, Bomberg H, Schuster S, Schäfers H-J, Groesdonk HV. Prognostic value of procalcitonin in patients after elective cardiac surgery: a prospective cohort study. Ann Intens Care 2016;6:116.

Lee YS, Koo KH, Kim HJ, et al. Synovial fluid biomarkers for the diagnosis of periprosthetic joint infection: a systematic review and meta-analysis. J Bone Joint Surg Am 2017;99:2077-2084.

Li X, Wang X, Li S, Yan J, Li D. Diagnostic value of procalcitonin on early postoperative infection after pediatric cardiac surgery. Pediatr Crit Care Med 2017;18:420-428.

Pittet D, Rangel-Frausto S, Li N, et al. Systemic inflammatory response syndrome, sepsis, severe sepsis and septic shock: incidence, morbidities and outcomes in surgical ICU patients. Intensive Care Med 1995;21:302-309.

Russell CD, Parajuli A, Gale HJ, et al. The utility of peripheral blood leucocyte ratios as biomarkers in infectious diseases: a systematic review and meta-analysis. J Infect 2019;78:339-348.

Spaziante M, Ceccarelli G, Al Moghazi S, Alessandri F, Venditti M. Specific dynamic of serum procalcitonin in critically ill patients affected by Gram-negative bacilli septic thrombophlebitis. Crit Care 2018;22:178.

Tan M, Lu Y, Jiang H, Zhang L. The diagnostic accuracy of procalcitonin and C-reactive protein for sepsis: a systematic review and meta-analysis. J Cell Biochem 2019;120:5852-5859.

Torres A, Niederman MS, Chastre J, et al. International ERS/ESICM/ ESCMID/ALAT guidelines for the management of hospital-acquired pneumonia and ventilator-associated pneumonia. Eur Respir J 2017;50:1700582.

Vos RJ, Van Putte BP, Kloppenburg GTL. Prevention of deep sternal wound infection in cardiac surgery: a literature review. J Hosp Infect 2018;100:411-420.

Wallgren UM, Antonsson VE, Castrén MK, Kurland L. Longer time to antibiotics and higher mortality among septic patients with non-specific presentations-a cross sectional study of Emergency Department patients indicating that a screening tool may improve identification. Scand J Trauma Resusc Emerg Med 2016;24:1.

Weiss SL, Fitzgerald JC, Balamuth F, et al. Delayed antimicrobial therapy increases mortality and organ dysfunction duration in pediatric sepsis. Crit Care Med 2014;42:2409-2417. 